\title{
GO-based Functional Dissimilarity of Gene Sets
}

\author{
Norberto Díaz-Díaz and Jesús S Aguilar-Ruiz
}

\begin{abstract}
Background: The Gene Ontology (GO) provides a controlled vocabulary for describing the functions of genes and can be used to evaluate the functional coherence of gene sets. Many functional coherence measures consider each pair of gene functions in a set and produce an output based on all pairwise distances. A single gene can encode multiple proteins that may differ in function. For each functionality, other proteins that exhibit the same activity may also participate. Therefore, an identification of the most common function for all of the genes involved in a biological process is important in evaluating the functional similarity of groups of genes and a quantification of functional coherence can helps to clarify the role of a group of genes working together.

Results: To implement this approach to functional assessment, we present GFD (GO-based Functional Dissimilarity), a novel dissimilarity measure for evaluating groups of genes based on the most relevant functions of the whole set. The measure assigns a numerical value to the gene set for each of the three GO sub-ontologies.

Conclusions: Results show that GFD performs robustly when applied to gene set of known functionality (extracted from KEGG). It performs particularly well on randomly generated gene sets. An ROC analysis reveals that the performance of GFD in evaluating the functional dissimilarity of gene sets is very satisfactory. A comparative analysis against other functional measures, such as $\mathrm{GS}^{2}$ and those presented by Resnik and Wang, also demonstrates the robustness of GFD.
\end{abstract}

\section{Background}

The Gene Ontology (GO) [1] is a cross-species, controlled vocabulary describing three major functional characteristics of gene products: molecular function, cellular component and biological process. The information is structured as a directed acyclic graph for each subontology. Each node in the graph represents a class of genes identified by a GO-term. Each edge represents the relationship between the terms it connects, which can be "is a", "is a part of", or "regulates", meaning that a child class is either a part of the parent, is a more specific example of the parent class or is regulated (positively or negatively) by the parent, respectively.

The GO provides a controlled vocabulary for describing gene product functions and can be used to evaluate the functional coherence of gene sets. There are two major approaches for analyzing GO-based gene annotations: enrichment tools and semantic similarity measures.

\footnotetext{
* Correspondence: ndiaz@upo.es

School of Engineering, Pablo de Olavide University, Seville, Spain
}

\section{Enrichment tools}

Enrichment tools are used to determine the common features of a set of genes by examining annotations and finding GO-terms that they share to a significant extent. For example, the To-Go java navigation tool [2] allows users to navigate the $\mathrm{GO}$ with various kinds of queries. There are also enrichment tools that provide ontological analyses with different statistical models, including the hypergeometric, binomial, Pearsons's chi-squared and Fisher's exact tests [3]. GeneTools [4] is a web service that provides access to several databases such as UniGene [5], Entrez Gene [6], Swiss-Prot [7] and Gene Ontology. It includes a tool for visualisation and statistical hypothesis testing to assess the similarity of GO-term annotations in different gene lists. The local graph structure of GO hierarchy is available from GOLEM (Gene Ontology Local Exploration Maps) [8]. It also supports rapid analysis of an input list of genes to find enriched GO terms. FuncAssociate [9], takes a list of genes as an input and indicates whether a significant number of the genes share a certain GO term. Based on the same concept of GO

\section{() Biomed Central}

(C) 2011 Díaz-Díaz and Aguilar-Ruiz; licensee BioMed Central Ltd. This is an Open Access article distributed under the terms of the Creative Commons Attribution License (http://creativecommons.org/licenses/by/2.0), which permits unrestricted use, distribution, and reproduction in any medium, provided the original work is properly cited. 
enrichment, the tools Bingo [10] and Ease (DAVID) [11] take a set of genes and identify the saturated terms.

\section{Semantic similarity measures}

In general, GO-based enrichment tools are used to analyse GO term in large-scale gene sets. However, while they all determine whether an observed number of GO annotations in a set is significant, they lack a quantitative similarity measure that would allow for a complete comparable analysis of gene sets or models produced by microarray analysis. Although many enrichment tools are used for analyzing microarray data and give a level of significance for the designed enrichment, they only inform about the data distribution and do not give information on the inherent relationship, which is critical at comparing sets of genes.

To address this issue, semantic similarity methods have been developed. This category comprises GO-term, geneproduct and gene-similarity approaches. GO-term similarity approaches have been presented by Couto et al. [12], Kriventseva et al. [13], and Lee et al. [14] and are based on measures [15-17] originally developed for other semantic taxonomies. These measures determine the similarity of two GO-terms using the information content for their lowest common ancestor. Guo et al. [18-20] evaluated these methods and showed that Resnik's method is better than the others in terms of correlating gene sequence similarities and gene expression profiles. del Pozo et al. [21] proposed a new method for quantifying functional distances between GO terms. Their method is based on the simultaneous occurrence of terms in the same set of Interpro [22] entries does not rely on the structure of the GO itself. In the same vein, Wang et al. [23] presented a method to encode a GOterm's semantics as a numeric value by aggregating the semantic contributions of ancestor terms. This proposal was used to develop a clustering tool to study the genes in pathways retrieved from the Saccharomyces Genome Database (SGD), and the clustering results showed that Wang's method is more consistent than Resnik's method.

The relationships of gene-products are also of interest to researchers. Until recently, the most common methods for measuring gene-product functional similarity were pairwise approaches based on GO-term. Lord et al. [24] were the first to apply this methodology, using the average of all pairwise similarities. The same approach was used by Liu et al. [25], Azuaje et al. [26] and Chagoyen et al. [27]. Lee et al. [14] and Guo et al. [28] used the maximum of all pairwise similarities; whereas Brameier and Wiuf [29] and Wu et al. [30] used a composite average in which only the best-matching term pairs are considered (best-match average). Tao et al. [31] proposed a variant, in which only those pairwise measures that exceed a threshold were considered. The performances of these pairwise-based measures were tested by $\mathrm{Xu}$ et al. [32] They concluded that the method based on the bestmatch average consistently gave the best performance out of all of the tests that were studied.

Other methods exist for measuring gene-product functional similarity that are not based on pairwise approaches. Lerman and Shakhnovich [33] presented several manifoldembedding techniques for computing distances between GO functional annotations and for estimating functional distances between protein domains. Likewise, Schlicker et al. $[34,35]$ proposed a method for measuring functional similarity by combining different ontologies to produce a single similarity score. Their method, named FunSim, is based on Schlicker's measure $\left(\operatorname{sim}_{R e l}\right)$, which combines both of the Lin and Resnik GO-term similarity measures.

Bastos et al. [36] proposed three different measures: $G O_{\text {ocurrence }}$ to measure the functional coherence of a list of gene products; $G O_{\text {score }}$ to indicate how well a cluster of genes has been functionally characterised; and $G O_{c e n t e r}$ to provide a measure of how many of the cluster's functional annotations are captured by the center of the cluster. Zheng and $\mathrm{Lu}$ [37] developed a measure to determine the overall functional coherence of a group of proteins by using the semantic similarity of the biomedical literature associated with the proteins.

The methods presented above measure the similarity of a pair of GO terms, a measurement that can be extended to a set of gene products or a pair of genes. The approaches that address the functional coherence of a gene set do not simply select the most common function found within the set. Instead, the gene set coherence is determined as a function of the similarity of all the pairs of genes within the set [23]. Recently, Ruths et al. [38] proposed a GObased measure of functional similarity for gene sets, named $\mathrm{GS}^{2}$. This measure quantifies the similarity of a set of genes by averaging their individual contributions. Each gene is compared to the other genes in the set by calculating how closely the gene's annotation match the annotations of the others. GS ${ }^{2}$ was compared to the GO pairwise measure of Wang et al. [23] by extending Wang's measure to average the contributions of all gene pairs. The comparison showed that $\mathrm{GS}^{2}$ generates results more quickly and with comparable quality. To our knowledge, $\mathrm{GS}^{2}$ was the first method used to determine the functional similarity of a gene set by using an entire set of genes. Later, Richards et al. [39] proposed another GO-based measure to evaluate the functional coherence of gene sets. These measures are based on the topological properties of graphs comprised of genes and their GO annotations, and they consider the enrichment of annotations and the relationships among annotations when determining the significance of functional coherence. Unlike our approach, the method of 
Richards et al. considers all of the annotations with equal weight. The reader is refered to reference [40] for a survey of semantic similarity measures.

Although these similarity measures have been used for different applications [40], such as comparing gene products with different functions or predicting gene product functions, they face a major limitation when confronted with genes that are involved in several functions. For such genes, the current tools give equal weight to all of the biological functions and it is not possible to single out the most relevant ones by considering the context of the other genes involved [3].

In this work, we propose a novel method for measuring gene set dissimilarity by weighting the most cohesive (common and specific) functions based on the global behaviour of the whole set of input genes. This measure, named GFD, is based on the Gene Ontology, and it assigns a numerical value to a gene set for each of the three GO ontologies.

\section{Method}

GFD is based on an adaptation of the GO-tree structure presented in [14]. The structure is used to develop a novel GO-term dissimilarity measure for use in calculating gene set dissimilarity. To our knowledge, this is the first report of a measure that evaluates a gene set by taking into account the most cohesive function found in the set. The method involves searching for the most specific function for each gene that is also similar to the other functions found in the gene set.

The methodology is outlined in Figure 1, which presents an example of a set of four genes. The calculation of GFD entails five consecutive steps, which are described below.

\section{First step: Gene Identification}

The first step consists of finding the representative of each input gene in the GO. Let us assume that the GO maps to $\Theta$ genes for a specific organism. Let $A$ be the set of genes to be evaluated. Each $g \in A$ is searched for in $\Theta$, and if the search is unsuccessful, $g$ is transformed into a synonym $g$ ' by using the gene synonym information given in the GO annotation [41]. That is, the initial set of genes $A=\left\{g_{1}, \ldots\right.$, $\left.g_{n}\right\}$ is transformed into $A^{\prime}=\left\{g_{1}^{\prime}, \ldots, g_{n^{\prime}}^{\prime}\right\}$, where each $g^{\prime}$ is present in $\Theta$. However, the gene is removed if no synonym exists. For instance, in Figure 1, genes $g 1$ and $g 3$ were found in the GO, gene $g 2$ was transformed into a synonym $\left(g 2^{\prime}\right)$, and gene $g 4$ was not found.

\section{Second step: Gene-function Identification}

The second step consists of identifying the function of the genes in a set. Each gene is transformed into the different proteins encoded by the gene (the gene products) according to the Entrez Gene database [6]. Thus, a set of gene products $H(i)=\left\{g_{i} p_{1}, \ldots, g_{i} p_{m}\right\}$ is associated with each $g_{i}^{\prime} \in A^{\prime}$. Continuing with the previous example, $g_{1}$ encodes the $g_{1} p_{1}$ protein, $g_{2}$ encodes the $g_{2} p_{1}$ protein, and $g_{3}$ encodes $g_{3} p_{1}, g_{3} p_{2}$ and $g_{3} p_{3}$.

\section{Third step: Gene-product Filtering}

In this step, each gene function is filtered by the three GO domains. The proteins chosen in the previous step are removed from those domains in which they are not involved, and are otherwise selected. Thus, in Figure 1, the $g_{1} p_{1}$ protein, encoded by gene $g_{1}$, takes part in all three ontologies; the protein encoded by gene $g_{2}\left(g_{2} p_{1}\right)$ is presented in the Biological Process and Cellular Component ontologies; and gene $g_{3}$ is represented by protein $g_{3} p_{1}$ in the Molecular Function ontology, by the $g_{3} p_{1}$ and $g_{3} p_{2}$ proteins in the Biological Process, and by the $g_{3} p_{1}$ protein in the Cellular Component ontology. Once the input genes have been transformed into their biological functionalities and these have been filtered through each domain, the next steps must be repeated for the three ontologies, vielding three different results (one per ontology). For the sake of clarity, we will only consider the Biological Process ontology in the following descriptions and examples.

\section{Fourth step: Gene-product Annotation Search}

For each ontology, the annotations of each protein are examined. A single protein can be associated with or located in one or more cellular components, it is active in one or more biological processes, during which it might perform several molecular functions. This feature is accounted for in the GO: each functional annotation is identified by a unique $G O$ term.

In Figure 1, the annotations of each protein in the Biological Process ontology are depicted. For each $g_{i} p_{j}$, a set of GO terms is obtained, i.e., $H(i, j)=\left\{g_{i} p_{j} t_{1}, \ldots\right.$, $\left.g_{i} p_{j} t_{q}\right\}$. For example, protein $g_{1} p_{1}$ has two different terms in the Biological Process domain. Both functionalities are used for the $g_{1} p_{1} t_{1}$ and $g_{1} p_{1} t_{2}$ GO terms.

\section{Fifth step: Gene-product Functionality}

At this stage, each functional annotation in the GO has been indentified. The GO directed acyclic graph (DAG) is used, but only the "is a" relationships are considered. Our approach does not use the "part of" relationships for three reasons: a) we would like to compare results among the three domains, and the Molecular Function ontology does not have the "part of" relationship; b) the "part of" relationship is used in the biological process ontology when the child node is an instance of only a portion of the parent process; c) the three ontologies are now "is a" complete, meaning that every term has a path to the root node that passes solely through "is a" relationships. In Figure 1, the $g_{1} p_{1} t_{2}$ GO term is identical to the $g_{3} p_{2} t_{2}$ term. Therefore, both functions are located in the same node of the 


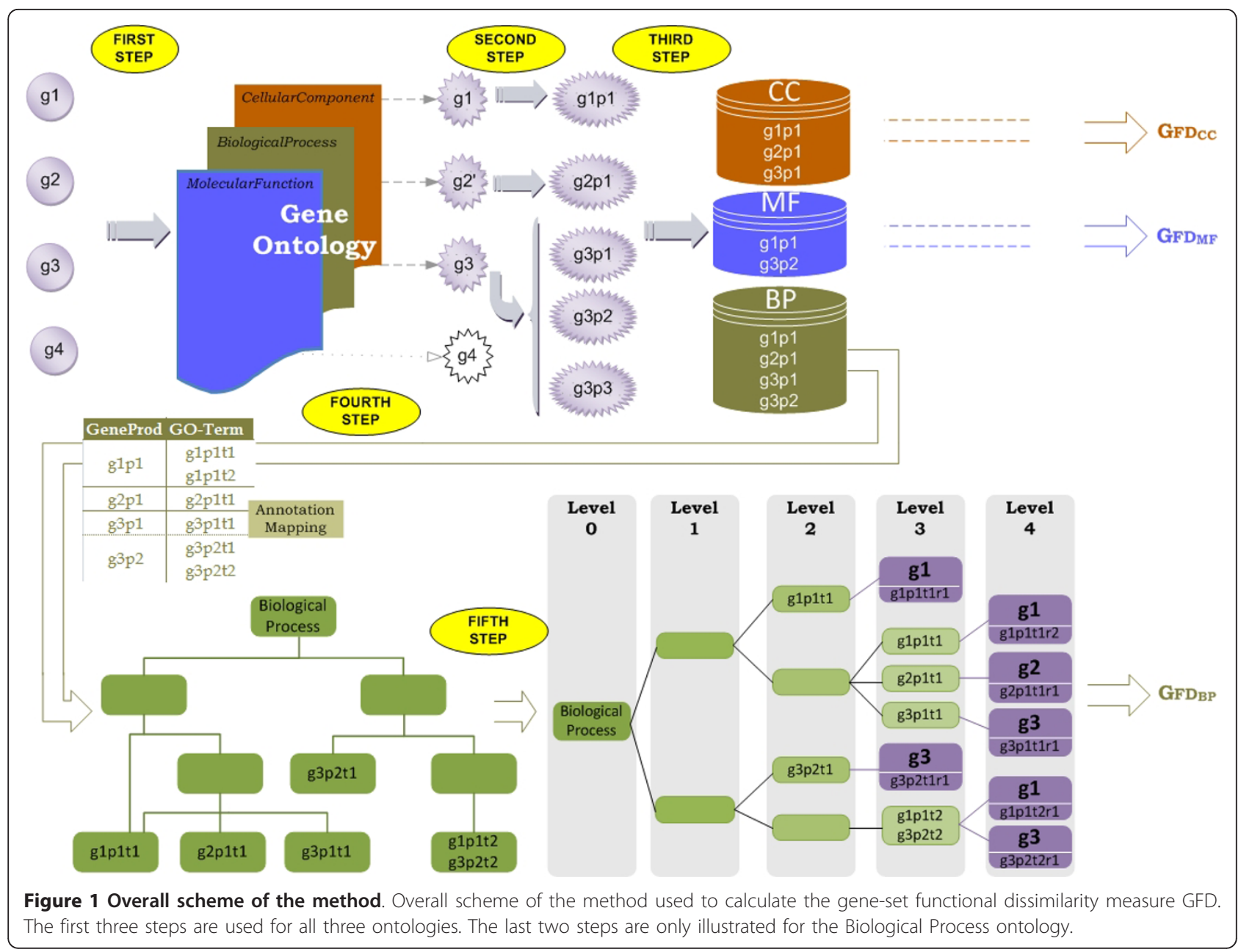

GO DAG. Next, the retrieved information is transformed into a tree structure (GO tree). A node will be present in the GO tree for each path that exists in the DAG from that node to the root. For instance, in Figure $1, g_{1} p_{1} t_{1}$ has two ways to reach the Biological Process root node, so this node is duplicated in the resulting GO tree.

Once the tree structure is built, the input genes are added to the GO tree as leaf nodes. These node positions designate the functional annotations found among the gene set. Each leaf node position is determined according to both the GO term and the protein product of the gene. A gene can be present in different leaves, which are different representations of the gene from different domains. Each GO-term $g_{i} p_{j} t_{k}$ will have a number of representations in the GO (the path from the GO term to the root), as it can be present in different places within the GO tree. This set of representations is denoted by $H(i, j, k)=\left\{g_{i} p_{j} t_{k} r_{1}, \ldots, g_{i} p_{j} t_{k} r_{s}\right\}$, where $r_{1} \ldots r_{s}$ denote the representations of term $g_{i} p_{j} t_{k}$.

After the GO tree is constructed, the input genes can be evaluated. At this point, the initial information $A=$ $\left\{g_{1}, \ldots, g_{4}\right\}$ has been transformed into three representations of $g_{1}$, one representation of $g_{2}$, and three representations of $g_{3}$, each of which is located in a structure that also provides information itself. The gene set functional measure GFD, which is described in detail below, is based on the gene-representation similarity and is supported by the GO-tree structure.

\section{Gene-Representation Functional Dissimilarity}

Let $r_{\alpha}$ and $r_{\beta}$ be two gene representations. The dissimilarity between them is given by:

$$
R\left(r_{\alpha}, r_{\beta}\right)=\frac{\operatorname{length}\left(r_{\alpha}, r_{\beta}\right)}{\operatorname{depth}\left(r_{\alpha}\right)+\operatorname{depth}\left(r_{\beta}\right)}
$$

where length $\left(r_{\alpha}, r_{\beta}\right)$ denotes the minimum number of nodes separating $r_{\alpha}$ from $r_{\beta}$ in the GO tree (i.e., the number of nodes in the path from $r_{\alpha}$ to $r_{\beta}$ ) and depth indicates the level of representation in the GO tree. From a biological point of view, length indicates the functional relationship of the two GO terms, whereas depth 
indicates the level of specificity of the representation. Thus, the measure penalises gene-representation pairs that are widely separated, and it rewards specialisation. This measure provides values between 0 and 1 , where values close to 0 mean "similar", and values near 1 mean "dissimilar".

Two gene representations, $r_{\alpha}$ and $r_{\beta}$, present the best similarity when they share the same parent $\left(\operatorname{length}\left(r_{\alpha}, r_{\beta}\right)=\right.$ $1)$ and their depths are the maximum $\left(\operatorname{depth}\left(r_{\alpha}\right)=\right.$ depth $\left.\left(r_{\beta}\right)=k\right)$. In this case, their functional dissimilarity is:

$$
R\left(r_{\alpha}, r_{\beta}\right)=\frac{1}{k+k} \approx 0
$$

In contrast, the worst case occurs when two gene representations are low in the GO tree $\left(\operatorname{depth}\left(r_{\alpha}\right)=\right.$ $\operatorname{depth}\left(r_{\beta}\right)=k$ ), and they do not share any ancestor node other than the root node (length $\left.\left(r_{\alpha}, r_{\beta}\right)=k+k-1\right)$ :

$$
R\left(r_{\alpha}, r_{\beta}\right)=\frac{2 k-1}{2 k} \approx 1
$$

For example,

$$
\begin{aligned}
& R\left(g_{1} p_{1} t_{2} r_{1}, g_{3} p_{2} t_{2} r_{1}\right)=\frac{1}{4+4}=0.125 \\
& R\left(g_{1} p_{1} t_{1} r_{2}, g_{3} p_{2} t_{2} r_{1}\right)=\frac{7}{4+4}=0.875
\end{aligned}
$$

For this set of genes, the minimum and maximum values for $R$ are 0.125 and 0.875 , respectively.

\section{Functional Dissimilarity Measure}

The functional dissimilarity is based on the gene-representation dissimilarity described above. Our approach extrapolates the gene-representation dissimilarity measure to evaluate gene set homogeneity. Let $A$ be a set of genes $\left\{g_{1}, g_{2}, \ldots, g_{n}\right\}$. The representation set for a gene $g_{i}$ is given by $T\left(g_{i}\right)$, as shown in Equation 2, (see $H(i, j, k$ ) in the fifth step).

$$
T\left(g_{i}\right)=\bigcup_{\substack{j \in H(i) \\ k \in H(i, j)}} H(i, j, k)
$$

The Cartesian product $P(A)=T\left(g_{1}\right) \times T\left(g_{2}\right) \times \ldots \times T\left(g_{n}\right)$ defines the set of all possible sets of representations. The dissimilarity $S$ of a representation set $p \in P$ is given by Equation 3, where $R$ is the dissimilarity of two gene representations as calculated by Equation 1 . Note that $|p|=|A|$.

$$
S(p)=\frac{1}{\left(\begin{array}{c}
|p| \\
2
\end{array}\right)} \sum_{\forall \delta, \gamma|0<\delta<\gamma \leq| p \mid} R(p[\delta], p[\gamma])
$$

Finally, the GO-based functional dissimilarity, GFD, is the minimum dissimilarity for all possible representation sets for a given set of genes $A$.

$$
\operatorname{GFD}(A)=\min _{p \in P(A)} S(p)
$$

In Figure 1 there are seven representations (three for $T$ $\left(g_{1}\right)$, one for $T\left(g_{2}\right)$, and three for $\left.T\left(g_{3}\right)\right)$, which can generate nine possible sets of representations $(3 \times 1 \times 3)$, so $|P(A)|=9$. There are two optimal representations for $g_{1}$ and another two for $g_{3}$, which yield four possible optimal configurations. However, there is only one optimal functional combination according to the cohesive function of all genes. By randomly selecting, we could obtain the worst case $\left(S\left(g_{1} p_{1} t_{1} r_{1}, g_{2} p_{1} t_{1} r_{1}, g_{3} p_{2} t_{2} r_{1}\right)=0.768\right)$, in contrast to the best case $\left(S\left(g_{1} p_{1} t_{1} r_{2}, g_{2} p_{1} t_{1} r_{1}, g_{3} p_{1} t_{1} r_{1}\right)=\right.$ 0.428 ).

It is worth noting that our approach does not select the best GO term for each gene individually; instead, it searches for the most common and specific function for the whole set of genes. In this sense, GFD is quite different from $\mathrm{GS}^{2}$, because it only selects one function per gene (the most globally cohesive function), whereas $\mathrm{GS}^{2}$ considers all of the gene functions.

\section{Results and Discussion ROC analysis}

The performance of our approach was tested by comparing it to three different measures: an information content-based measure (Resnik [17]); a hybrid (node- and edge-based) measure (Wang [23]); and $\mathrm{GS}^{2}$ [38], the first measure reported to efficiently evaluate sets of genes instead of pairs of genes or GO terms. Both Resnik and Wang's measures for terms were calculated using their implementations in Bioconductor [42] and extrapolated to gene sets using the best-match average approach. The $\mathrm{GS}^{2}$ source was downloaded from the website referenced in [38].

ROC analyses have been widely used in the literature [32] because they can be used to score the performance of classifiers and rankers as a trade-off between sensitivity or as a true positive rate and false positive rate. In addition, the area under the ROC curve is presented, as it provides information about the level of randomness of the approach.

Two data sets were used: sets with and without functional coherence. Both data sets were generated according to the information stored in KEGG. KEGG[43] is a database of biological systems that integrates genomic, chemical and systemic function information. This database offers genomic information about several hundred organisms, from which we selected Saccharomyces cerevisiae (SCE). All of the metabolic pathways from SCE were used as examples of gene sets with functional coherence. A cluster of genes was associated with each pathway. The data set without functional coherence was designed to be the same size as the functional coherence, but the genes 
within the clusters were randomly generated. Thus, for each pathway, we have two gene clusters of size $k$ : one with genes involved in the same pathway and another with randomly generated genes.

ROC analysis was performed for the three GO ontologies. In particular, the GFD, Resnik and Wang methods were compared for the three ontologies, whereas GS was only used for the Biological Process ontology because this measure can-not provide values for the other two ontologies. The ROC curve was plotted over the interval $[0,1]$ with increments of 0.01 , as illustrated in Figure 2. The area under the ROC curve (AUC) is enclosed in brackets.

GFD shows similar and satisfactory behaviour for the three ontologies. The Resnik and Wang methods perform differently. For the Biological Process ontology, only Wang's approach performs worse than expected, due to its false positive rate. The AUC is above 0.90 for most measures, except for Wang's, which seems to be random (below 0.5). For the Molecular Function ontology, our approach is excellent, with an AUC of about 0.98, which is much greater than that of Resnik (0.65) or Wang's approach(0.17). For the Cellular Component ontology, the performances of the three measures are similar.

Although a biological process is not equivalent to a pathway, these concepts are very similar. For example, Cell cycle pathway (sce:04111) is directly related to "mitotic cell cycle" GO-term according to the information stored in KEGG. Thus, genes within the same pathway must be similar in the Biological Process ontology. However, those genes do not have to be similar in all cases under the Cellular Component ontology since they can be located in different places of cell for some pathways. For example, Cell cycle genes related to transcription are located in the nucleus while those related to translation are in the ribosome. Hence, the results obtained in this ontology are not sufficiently consistent in order to compare the performance of the different approaches. Finally, genes in the same pathway also have to be similar in the Molecular Function ontology. This ontology describes types of activities, some of which are present in the pathway describing the process. This is crucial in our study since our approach select the most cohesive function among the genes. In contrast, Wang and Resnik approaches are based on the best-match average where the same functionality may not neccessarily be selected to compute the overall similarity of the set of genes. This will cause a high false positive rate (FPR). GFD only uses one GO-term to evaluate the similarity of a gene in
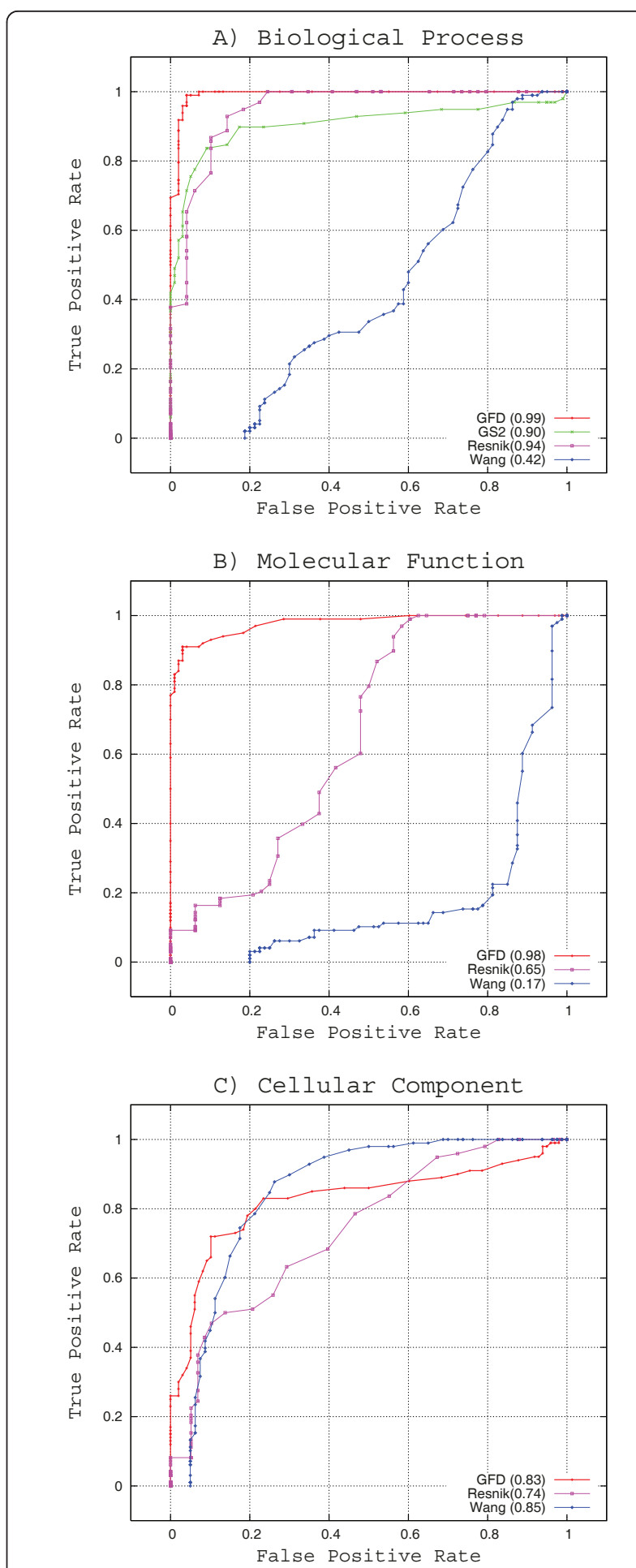

Figure 2 ROC Analysis. ROC analysis for the GFD, GS ${ }^{2}$, Wang and Resnik approaches, as applied to each of three GO ontologies. The area under the ROC curve is indicated in brackets. 
relation to the rest, while the other approaches can select different GO-terms to measure the similarity of a gene with regard to the other genes. This is the main reason for the poor performance of the Wang and Resnik in Molecular Function compaired to Biological Process. In general, the ROC analysis shows the robustness of GFD and demonstrates the effectiveness of the approach in evaluating the most cohesive functional annotation of a set of genes.

\section{Computational analysis}

The gene set functional coherence measure proposed here is based on calculating the dissimilarity of all possible input gene-representation combinations. If the input set has $n$ genes and each gene encodes $p$ gene products, then each gene product supports $t \mathrm{GO}$ terms in each ontology, and the average number of gene representations per GO term is $r$. The computational order of the similarity measure is:

$$
T(n) \in \Theta\left((p \times t \times r)^{n} \times n^{2}\right)=\Theta\left(K^{n} \times n^{2}\right)
$$

where $K=p \times t \times r$ is the number of gene representations per gene. $K^{n}$ is the number of different gene representation sets for each input gene. The number- of generepresentation pairs is $n^{2}$. Consequently, the exhaustive calculation of GFD has a high computational complexity, making it intractable for large data sets. As the homogeneity measure for any set of genes should be calculated in an efficient way, we introduce a heuristic technique based on the Voronoi Diagram concept [44] that reduces the complexity from exponential to polynomial order. For each node in the GO tree, the nearest representation of each gene for that node is obtained (according to $\mathrm{R}$ in Eq. 1). Thus $T\left(g_{i}\right)$ (see Eq. 2) represents the set with a unique representation for each node (the nearest from gene $i$ to the node). Once the nearest representation of each gene is found for each node, the dissimilarity values of the nodes are calculated (Eq. 3). Finally, the smallest value found for $S$ is used as the GFD value.

To explore the effect of heuristics on the computational cost, we analysed the well-known cell cycle pathway (sce:04111) from Saccharomyces cerevisiae. To evaluate this set, which contains 125 genes, it is necessary to consider their 909 annotations. These annotations have 10, 410 representations, which generate $10^{216.56}$ combinations. Our approach can evaluate these combinations in only 20 seconds (running on a laptop workstation). Similarly, to evaluate the 125 randomly chosen genes, $10^{149.46}$ combinations were generated and evaluated in 29 seconds. Table 1 shows the computational cost of the five largest sets, together with relevant information about the number of annotations, representations and combinations for each set of genes (the first row depicts the sets of genes obtained from metabolic pathways, and the second row shows the sets of randomly chosen genes).

The performance of the approach, in terms of the influence of the heuristics on the quality of results, was also analysed. Varying the number of children per node (from 3 to 4 ) and the number of input genes (from 3 to $10)$ and randomly generating 100 different trees for each setting, 1600 trees were considered for analysis with and without the heuristics. The use of the heuristic algorithm produced slightly different results in $2.5 \%$ of the cases ( 44 trees), and the average relative error was 0.0005 , indicating that the reduction of computational cost does not significantly affect the quality of the results.

\section{Conclusions}

We have introduced a functional dissimilarity measure for gene sets, named GFD (GO-based Functional Dissimilarity) that selects the most cohesive function from a set of input genes. GFD was compared to the most relevant techniques: GS ${ }^{2}$ and the methods of Wang et al. and Resnik et al. Comparisons were conducted for

Table 1 Computational analysis

\begin{tabular}{|c|c|c|c|c|c|}
\hline Pathway & Genes & Annotations & Representations & Combinations $\left(\log _{10}\right)$ & Time (sec) \\
\hline \multirow[t]{2}{*}{ sce01100 } & 645 & 2544 & 72354 & 1131.73 & 1126 \\
\hline & & 2046 & 23578 & 727.49 & 645 \\
\hline \multirow[t]{2}{*}{ sce01110 } & 235 & 1005 & 26745 & 430.06 & 114 \\
\hline & & 716 & 10502 & 277.62 & 108 \\
\hline \multirow[t]{2}{*}{ sce03008 } & 157 & 312 & 4090 & 208.11 & 3 \\
\hline & & 557 & 7883 & 189.89 & 41 \\
\hline \multirow[t]{2}{*}{ sce04113 } & 127 & 884 & 8850 & 204.08 & 19 \\
\hline & & 450 & 4917 & 152.66 & 33 \\
\hline \multirow[t]{2}{*}{ sce04111 } & 125 & 909 & 10410 & 216.56 & 20 \\
\hline & & 461 & 5801 & 149.46 & 29 \\
\hline
\end{tabular}

The five Saccharomyces cerevisiae pathways with the highest number of known genes. For each set of genes (each pathway) the upper row represents real data and the lower row illustrates the pseudorandomly generated data. 
two different data sets: one based on KEGG pathways and another that was pseudorandomly generated.

To demonstrate the robustness of the method, an ROC analysis was performed for the three GO ontologies to analyse the discriminatory power of the dissimilarity mesures and their sensitivity. In general, GFD is much more accurate for the Molecular Function ontology, and it is equivalent to the Resnik and GS $^{2}$ methods for the other two ontologies. The area under the ROC curve also shows good performance for both the Biological Process and Molecular Function ontologies (0.99 and 0.98 , respectively).

\section{Acknowledgements}

This research was partially supported by the Ministry of Science and Innovation, projects TIN2007-68084-C02-00 and PCI2006-A7-0575, and by the Junta de Andalucia, projects P07-TIC-02611 and TIC-200.

ND thanks Jeffrey Chuang and Kourosh Zarringhalam for helpful discussions.

\section{Authors' contributions}

ND conceived the method, designed and implemented the algorithm, and conducted the study. JAR lead the project and participated in writing the manuscript. All authors read, edited and approved the final manuscript.

Received: 26 November 2010 Accepted: 1 September 2011 Published: 1 September 2011

\section{References}

1. Ashburner M, Ball CA, Blake JA, Botstein D, Butler H, Cherry JM, Davis AP, Dolinski K, Dwight SS, Eppig JT, Harris MA, Hill DP, Issel-Tarver L, Kasarskis A, Lewis S, Matese JC, Richardson JE, Ringwald M, Rubin GM, Sherlock G: Gene ontology: tool for the unification of biology. The Gene Ontology Consortium. Nature Genetics 2000, 25:25-29.

2. Yu U, Choi Y, Choi J, Kim S: TO-GO: a Java-based Gene Ontology navigation environment. Bioinformatics 2005, 21(17):3580-3581.

3. Khatri $P$, Drăghici $S$ : Ontological analysis of gene expression data: current tools, limitations, and open problems. Bioinformatics 2005, 21(18):3587-3595.

4. Beisvag V, Junge FK, Bergum H, Jolsum L, Lydersen S, Gunther CC, Ramampiaro H, Langaas M, Sandvik AK, Laegreid A: GeneTools- application for functional annotation and statistical hypothesis testing. BMC Bioinformatics 2006, 7:470.

5. Pontius J, Wagner $L$, Schuler G: UniGene: a unified view of the transcriptome. The NCBI Handbook. Bethesda (MD): National Center for Biotechnology Information 2003.

6. Maglott D, Ostell J, Pruitt K, Tatusova T: Entrez Gene: gene-centered information at NCBI. Nucleic Acids Research 2005, , 33 Database: D54-D58.

7. Bairoch A, Apweiler R: The SWISS-PROT protein sequence database and its supplement TrEMBL in 2000. Nucleic Acids Research 2000, 28:45-48.

8. Sealfon RS, Hibbs MA, Huttenhower C, Myers CL, Troyanskaya OG: GOLEM: an interactive graph-based gene-ontology navigation and analysis tool. BMC Bioinformatics 2006, 7:443.

9. Berriz G, King O, Bryan B, Sander C, Roth F: Characterizing gene sets with FuncAssociate. Bioinformatics 2003, 19:2502-2504[http://lama.med.harvard. edu/Software.html].

10. Maere S, Heymans K, Kuiper M: BiNGO: a Cytoscape plugin to assess overrepresentation of Gene Ontology categories in Biological Networks. Bioinformatics 2005, 21(16):3448-3449[http://dx.doi.org/10.1093/ bioinformatics/bti551].

11. Huang DWaW, Sherman BT, Tan Q, Kir J, Liu D, Bryant D, Guo Y, Stephens R, Baseler MW, Lane HC, Lempicki RA: DAVID Bioinformatics Resources: expanded annotation database and novel algorithms to better extract biology from large gene lists. Nucleic acids research 2007, , 35 Web Server: gkm415+[http://dx.doi.org/10.1093/nar/gkm415].

12. Couto F, Silva M, Coutinho P: Implementation of a Functional Semantic Similarity Measure between Gene-Products. DI/FCUL TR 03-29, Department of Informatics, University of Lisbon 2003 [http://docs.di.fc.ul.pt/jspui/handle/ 10455/2935].

13. Kriventseva E, Fleischmann W, Apweiler R: CluSTr: a database of Clusters of SWISS-PROT+TrEMBL proteins. Nucelic Acids Res 2001, 29:33-36.

14. Lee $\mathrm{SG}$, Hur JU, Kim YS: A graph-theoretic modeling on GO space for biological interpretation of gene clusters. Bioinformatics 2004, 20(3):381-388.

15. Jiang JJ, Conrath DW: Semantic Similarity Based on Corpus Statistics and Lexical Taxonomy. International Conference Research on Computational Linguistics 1997, 19-33.

16. Lin D: An Information-Theoretic Definition of Similarity. Proceedings of the 15th International Conference on Machine Learning, Morgan Kaufmann, San Francisco, CA 1998, 296-304.

17. Resnik P: Semantic Similarity in a Taxonomy: An Information-Based Measure and its Application to Problems of Ambiguity in Natural Language. Journal of Artificial Intelligence Research 1999, 11:95-130.

18. Guo X, Liu R, Shriver CD, Hu H, Liebman MN: Assessing semantic similarity measures for the characterization of human regulatory pathways. Bioinformatics 2006, 22(8):967-973

19. Sevilla JL, Segura V, Podhorski A, Guruceaga E, Mato JM, Martinez-Cruz LA, Corrales FJ, Rubio A: Correlation between Gene Expression and GO Semantic Similarity. IEEE/ACM Trans Comput Biol Bioinformatics 2005, 2(4):330-338.

20. Wang H, Azuaje F, Bodenreider O, Dopazo J: Gene expression correlation and gene ontology-based similarity: an assessment of quantitative relationships. Proceedings of the 2004 IEEE Symposium on Computational Intelligence in Bioinformatics and Computational Biology (CIBCB'2004) 2004, 25-31.

21. Pozo AD, Pazos F, Valencia A: Defining functional distances over Gene Ontology. BMC Bioinformatics 2008, 9:50-65.

22. Hunter $S$, Apweiler $R$, Attwood TK, Bairoch A, Bateman A, Binns D, Bork P, Das U, Daugherty L, Duquenne L, Finn RD, Gough J, Haft D, Hulo N, Kahn D, Kelly E, Laugraud A, Letunic I, Lonsdale D, Lopez R, Madera M, Maslen J, McAnulla C, McDowall J, Mistry J, Mitchell A, Mulder N, Natale D, Orengo C, Quinn AF, Selengut JD, Sigrist CJA, Thimma M, Thomas PD, Valentin F, Wilson D, Wu CH, Yeats C: InterPro: the integrative protein signature database. Nucl Acids Res 2009, 37(suppl 1):D211-215.

23. Wang JZZ, Du Z, Payattakool R, Yu PSS, Chen CFF: A New Method to Measure the Semantic Similarity of GO Terms. Bioinformatics 2007 , 23(10):1274-1281.

24. Lord PW, Stevens RD, Brass A, Goble CA: Semantic similarity measures as tools for exploring the gene ontology. Pac Symp Biocomput 2003, 601-612 [http://view.ncbi.nlm.nih.gov/pubmed/12603061]

25. Liu H, Hu ZZ, Wu CH: DynGO: a tool for visualizing and mining of Gene Ontology and its associations. BMC bioinformatics 2005, 6: [http://dx.doi. org/10.1186/1471-2105-6-201].

26. Azuaje F, Al-Shahrour F, Dopazo J: Ontology-Driven Approaches to Analyzing Data in Functional Genomics. Methods in Molecular Biology 2005, 316:67-86.

27. Chagoyen M, Carazo J, Montano AP: Assessment of protein set coherence using functional annotations. BMC Bioinformatics 2008, 9:444+[http://dx. doi.org/10.1186/1471-2105-9-444]

28. Guo X: Gene Ontology-based Semantic Similarity Measures. World Wide Web electronic publication 2008 [http://www.bioconductor.org/packages/2.2/ bioc/vignettes/SemSim/inst/doc/SemSim.pdf].

29. Brameier M, Wiuf C: Co-clustering and visualization of gene expression data and gene ontology terms for Saccharomyces cerevisiae using selforganizing maps. J of Biomedical Informatics 2007, 40:160-173[http://portal. acm.org/citation.cfm?id=1230523]

30. Wu H, Su Z, Mao F, Olman V, Xu Y: Prediction of functional modules based on comparative genome analysis and Gene Ontology application. Nucleic Acids Research 2005, 33(9):2822-2837[http://dx.doi.org/10.1093/nar/ gki573].

31. Tao Y, Sam L, Li J, Friedman C, Lussier YA: Information theory applied to the sparse gene ontology annotation network to predict novel gene function. Bioinformatics 2007, 23(13):i529-538[http://dx.doi.org/10.1093/ bioinformatics/btm195].

32. $\mathrm{Xu} \mathrm{T,} \mathrm{Du} \mathrm{L,} \mathrm{Zhou} \mathrm{Y:} \mathrm{Evaluation} \mathrm{of} \mathrm{GO-based} \mathrm{functional} \mathrm{similarity}$ measures using $S$. cerevisiae protein interaction and expression profile data. BMC Bioinformatics 2008, 9:472+[http://dx.doi.org/10.1186/1471-21059-472]. 
33. Lerman G, Shakhnovich BE: Defining functional distance using manifold embeddings of gene ontology annotations. Proceedings of the National Academy of Sciences of the United States of America 2007, 104(27):11334-11339

34. Schlicker A, Domingues FS, Rahnenfuhrer J, Lengauer T: A new measure for functional similarity of gene products based on Gene Ontology. BMC Bioinformatics 2006, 7:302+.

35. Schlicker A, Albrecht M: FunSimMat: a comprehensive functional similarity database. Nucl Acids Res 2008, 36 Database: D434-D439.

36. Bastos H, Faria D, Pesquita C, Falcão AO: Using GO terms to evaluate protein clustering. BioOntologies SIG at ISMB/ECCB - 15th Annual International Conference on Intelligent Systems for Molecular Biology (ISMB) 2007 [http://hdl.handle.net/10455/6214].

37. Zheng $B, L u X$ : Novel metrics for evaluating the functional coherence of protein groups via protein semantic network. Genome Biology 2007, 8(7): R153+[http://dx.doi.org/10.1186/gb-2007-8-7-r153].

38. Ruths T, Ruths D, Nakhleh L: GS2: an efficiently computable measure of GO-based similarity of gene sets. Bioinformatics 2009, 25(9):1178-1184.

39. Richards AJ, Muller B, Shotwell M, Cowart LA, Rohrer B, Lu X: Assessing the functional coherence of gene sets with metrics based on the Gene Ontology graph. Bioinformatics 2010, 26(12):i79-i87[http://dx.doi.org/ 10.1093/bioinformatics/btq203]

40. Pesquita C, Faria D, Falcão AO, Lord P, Couto FM: Semantic Similarity in Biomedical Ontologies. PLoS Comput Biol 2009, 5(7):e1000443+.

41. Barrell D, Dimmer E, Huntley RP, Binns D, O'Donovan C, Apweiler R: The GOA database in 2009-an integrated Gene Ontology Annotation resource. Nucleic acids research 2009, , 37 Database: D396-403[http://dx.doi. org/10.1093/nar/gkn803].

42. Yu G, Li F, Qin Y, Bo X, Wu Y, Wang S: GOSemSim: an R package for measuring semantic similarity among $\mathrm{GO}$ terms and gene products. Bioinformatics 2010, 26(7):976-978[http://dx.doi.org/10.1093/bioinformatics/ bta064].

43. Kanehisa M, Goto S, Furumichi M, Tanabe M, Hirakawa M: KEGG for representation and analysis of molecular networks involving diseases and drugs. Nucleic acids research 2010, , 38 Database: D355-360.

44. Aurenhammer F, Klein R: In Voronoi Diagrams. Edited by: Sack J-R, Urrutia J. Amsterdan: Handbook of Computational Geometry. Elsevier Science Publishers B.V; 1999:

doi:10.1186/1471-2105-12-360

Cite this article as: Díaz-Díaz and Aguilar-Ruiz: GO-based Functional

Dissimilarity of Gene Sets. BMC Bioinformatics 2011 12:360.

\section{Submit your next manuscript to BioMed Central and take full advantage of:}

- Convenient online submission

- Thorough peer review

- No space constraints or color figure charges

- Immediate publication on acceptance

- Inclusion in PubMed, CAS, Scopus and Google Scholar

- Research which is freely available for redistribution

Submit your manuscript at www.biomedcentral.com/submit 